\title{
Ring transformation of 2-(haloalkyl)azetidines into 3,4-disubstituted pyrrolidines and piperidines
}

\author{
Willem Van Brabandt, Robin Van Landeghem and Norbert De Kimpe* \\ Department of Organic Chemistry, Faculty of Bioscience Engineering, \\ Ghent University, Coupure links 653, B-9000 Ghent, Belgium
}

norbert.dekimpe@UGent.be

\section{General experimental methods}

${ }^{1} \mathrm{H}$ NMR spectra were recorded at $300 \mathrm{MHz}$ with $\mathrm{CDCl}_{3}$ as solvent and tetramethylsilane as internal standard. ${ }^{13} \mathrm{C}$ NMR spectra were recorded at $75 \mathrm{MHz}$ with $\mathrm{CDCl}_{3}$ as solvent. Mass spectra were obtained with a mass spectrometer $(70 \mathrm{eV})$ using a GC-MS coupling (20 m glass capillary column, i.d. $0.53 \mathrm{~mm}$, He carrier gas) or were recorded using a direct inlet system $(70 \mathrm{eV})$. Diethyl ether and THF were dried by distillation over sodium benzophenone ketyl. All other solvents were used as received from the supplier.

\section{Synthesis of 4-(1-haloalkyl)azetidin-2-ones 1 and 4-(2-haloalkyl)azetidin-2-ones 10}

4-(1-Haloalkyl)azetidin-2-ones 1 and 4-(2-haloalkyl)azetidin-2-ones 10 were synthesised by $[2+2]$-cyclocondensation of the corresponding $\alpha$-halogenated or $\beta$-halogenated imines

\footnotetext{
* Tel: +0032 92645951, fax: +003292646243
} 
and ketenes (formed in situ from the corresponding acid chlorides by treatment with triethylamine) in benzene. ${ }^{14,15}$

\section{Reduction of 4-(1-haloalkyl)azetidin-2-ones 1 and 4-(2-haloalkyl)azetidin-2-ones 10 with chloroalane $\left(\mathrm{AlH}_{2} \mathrm{Cl}\right)$}

As an example, the synthesis of 3-benzyloxy-1-tert-butyl-2-(1-chloro-1methylethyl)azetidine 2a is described. To a solution of aluminum(III) chloride $(0.65 \mathrm{~g}$, $4.85 \mathrm{mmol})$ in $40 \mathrm{ml}$ of dry ether was added lithium aluminum hydride $(0.18 \mathrm{~g}, 4.85$ mmol) at $0^{\circ} \mathrm{C}$. The reaction mixture was stirred for 30 minutes at room temperature. 3Benzyloxy-1-tert-butyl-4-(1-chloro-1-methylethyl)azetidin-2-one 1a (0.5g, $1.62 \mathrm{mmol})$ in $10 \mathrm{ml}$ of dry ether was added dropwise at $0^{\circ} \mathrm{C}$. After four hours at $0^{\circ} \mathrm{C}(18$ hours in the synthesis of $\mathbf{3 a}$ and $\mathbf{3 b}$ ) water was added carefully to neutralise the residual hydride. After decantation of the solvent, the residue was extracted three times with $20 \mathrm{ml}$ of diethyl ether, and the combined organic fractions were dried $\left(\mathrm{MgSO}_{4}\right)$. After filtration and evaporation of the solvent 3-benzyloxy-1-tert-butyl-2-(1-chloro-1methylethyl)azetidine 2a was obtained in 63\% yield. Further purification was performed by flash chromatography. For additional spectral data see also ref. 16.

\section{3-Benzyloxy-2-(1-bromo-1-methylethyl)-1-tert-butylazetidine 2f}

White crystals, 61\% yield, TLC Rf 0.3 (petroleum ether/ethyl acetate 9/1), Mp. $58^{\circ} \mathrm{C}^{1} \mathbf{H}$ NMR $\left(300 \mathrm{MHz}, \mathrm{CDCl}_{3}\right): \delta 1.08(9 \mathrm{H}, \mathrm{s}), 1.78$ and $1.81(6 \mathrm{H}, 2 \times \mathrm{s}), 3.21(1 \mathrm{H}, \mathrm{d} \times \mathrm{d}, J=8.8$ $\mathrm{Hz}, J=7.2 \mathrm{~Hz}), 3.29(1 \mathrm{H}, \mathrm{d} \times \mathrm{d}, J=8.8 \mathrm{~Hz}, J=4.7 \mathrm{~Hz}), 3.73(1 \mathrm{H}, \mathrm{d}, J=7.4 \mathrm{~Hz}), 4.15$ 
$(1 \mathrm{H}, \mathrm{d} \times \mathrm{t}, J=7.2 \mathrm{~Hz}, J=4.7 \mathrm{~Hz}), 4.40(1 \mathrm{H}, \mathrm{d}, J=11.8 \mathrm{~Hz}), 4.48(1 \mathrm{H}, \mathrm{d}, J=11.8 \mathrm{~Hz})$, 7.22-7.35 (5H, m). ${ }^{13} \mathbf{C}$ NMR $\left(75 \mathrm{MHz}, \mathrm{CDCl}_{3}\right): \delta 26.1,29.6,29.7,51.7,52.5,72.4,71.2$, 71.5, 72.1, 127.4, 127.5, 128.3, 138.0. IR $\left(\mathrm{NaCl}, \mathrm{cm}^{-1}\right): v_{\max }=2970,2935,2871,1497$, 1456, 1364. MS $(70 \mathrm{eV}) \mathrm{m} / \mathrm{z}(\%): 296(100) ; 260\left(\mathrm{M}^{+}-\mathrm{Br}\right.$, 65). Anal. Calcd for $\mathrm{C}_{17} \mathrm{H}_{26} \mathrm{BrNO}$ : $\mathrm{C} 60.00 ; \mathrm{H} 7.70 ; \mathrm{N}$ 4.12. Found: C 60.23; H 7.55; N 4.01.

\section{4-Benzyloxy-1-tert-butyl-2,2-dimethylpyrrolidine 3a}

Colourless oil, $63 \%$ yield, TLC Rf $0.05\left(\mathrm{CH}_{2} \mathrm{Cl}_{2} / \mathrm{MeOH} 20 / 1\right) .{ }^{1} \mathbf{H}$ NMR $(300 \mathrm{MHz}$, $\left.\mathrm{CDCl}_{3}\right): \delta 1.03(9 \mathrm{H}, \mathrm{s}), 1.08(6 \mathrm{H}, 2 \times \mathrm{s}), 2.52-2.59(2 \mathrm{H}, \mathrm{m}), 2.67(1 \mathrm{H}, \mathrm{d} \times \mathrm{d}, J=9.5 \mathrm{~Hz}, J=$ $5.9 \mathrm{~Hz}), 3.09(1 \mathrm{H}, \mathrm{d} \times \mathrm{d}, J=9.5 \mathrm{~Hz}, J=6.2 \mathrm{~Hz}), 3.55(1 \mathrm{H}, \mathrm{t}, J=5.9 \mathrm{~Hz}), 4.49(1 \mathrm{H}, \mathrm{d}, J=$ $12.1 \mathrm{~Hz}), 4.57(1 \mathrm{H}, \mathrm{d}, J=12.1 \mathrm{~Hz}), 7.24-7.38(5 \mathrm{H}, \mathrm{m}) .{ }^{13} \mathrm{C}$ NMR $(75 \mathrm{MHz}, \mathrm{ref}=$ $\left.\mathrm{CDCl}_{3}\right): \delta 22.1,27.7,25.9,40.7,50.9,59.3,72.0,85.5,127.4,128.4,139.1$. IR $(\mathrm{NaCl}$, $\left.\mathrm{cm}^{-1}\right): v_{\max }=2968,2869,1728,1661,1606,1497,1455,1364 . \mathbf{M S}(70 \mathrm{eV}) \mathrm{m} / \mathrm{z}(\%): 262$ $\left(\mathrm{M}^{+}+1,100\right)$. Anal. Calcd for $\mathrm{C}_{17} \mathrm{H}_{27} \mathrm{NO}$ : $\mathrm{C} 78.11 ; \mathrm{H} \mathrm{10.41;} \mathrm{N} \mathrm{5.36.} \mathrm{Found:} \mathrm{C} 78.33 ; \mathrm{H}$ $10.29 ;$ N 5.51.

\section{1-Allyl-4-benzyloxy-2,2-dimethylpyrrolidine 3b}

Colourless oil, $48 \%$ yield, TLC Rf $0.15\left(\mathrm{CH}_{2} \mathrm{Cl}_{2} / \mathrm{MeOH} 20 / 1\right) .{ }^{1} \mathbf{H}$ NMR $(270 \mathrm{MHz}$, $\left.\mathrm{CDCl}_{3}\right): \delta 1.17(6 \mathrm{H}, \mathrm{s}), 2.76(1 \mathrm{H}, \mathrm{d}, J=10.6 \mathrm{~Hz}), 2.95(1 \mathrm{H}, \mathrm{d} \times \mathrm{d}, J=12.0 \mathrm{~Hz}, J=3.0$ $\mathrm{Hz}), 3.00(1 \mathrm{H}, \mathrm{d}, J=10.6 \mathrm{~Hz}), 3.41-3.48(2 \mathrm{H}, \mathrm{m}), 3.51(1 \mathrm{H}, \mathrm{d} \times \mathrm{d}, J=12.0 \mathrm{~Hz}, J=4.6$ Hz), 3.64-3.67 (1H, m), $4.34(1 \mathrm{H}, \mathrm{d}, J=11.9 \mathrm{~Hz}), 4.73(1 \mathrm{H}, \mathrm{d}, J=11.9 \mathrm{~Hz}), 5.28-5.37$ $(2 \mathrm{H}, \mathrm{m}), 5.97-6.10(1 \mathrm{H}, \mathrm{m}), 7.26-7.34(5 \mathrm{H}, \mathrm{m}) .{ }^{13} \mathrm{C}$ NMR $\left(67.8 \mathrm{MHz}, \mathrm{CDCl}_{3}\right): \delta$ 21.0, 26.3, 42.6, 56.9, 59.6, 64.0, 71.2, 121.7, 127.5, 128.4, 127.8, 130.6, 137.7). IR ( NaCl, 
$\left.\mathrm{cm}^{-1}\right): v_{\max }=3416,2963,2905,2870,2414,2203,1497,1469,1455,1364$. MS $(70 \mathrm{eV})$

m/z (\%): $246\left(\mathrm{M}^{+}+1,100\right)$. Anal. Calcd for $\mathrm{C}_{16} \mathrm{H}_{23} \mathrm{NO}$ : C 78.32; H 9.45; N 5.71. Found:

C 78.48; H 9.49; N 5.60.

Transformations of 2-(1-haloalkyl)azetidines 2 and 2-(2-haloalkyl)azetidines 10 towards pyrrolidines 4 and piperidines 12 and 13

Pyrrolidines 4 and piperidines 12 and 13 were synthesised starting from the corresponding azetidines 2 and $\mathbf{1 0}(4 \mathrm{mmol})$ using reaction conditions as described in table 1 . In the case of the use of sodium hydroxide $(40 \mathrm{mmol})$, little amounts of water (1 $\mathrm{ml})$ have to be added to DMSO $(30 \mathrm{ml})$ in order to dissolve sodium hydroxide. After the reaction, the solvent was evaporated in vacuo yielding crude $\mathbf{4 a}, \mathbf{4 b}$ or $\mathbf{1 2}$ when acetonitrile was used as solvent. When DMSO was used as solvent, after addition of water, the reaction mixture was extracted three times with diethyl ether $(20 \mathrm{ml})$ and the

combined organic fractions were washed three times with water $(20 \mathrm{ml})$. After drying $\left(\mathrm{MgSO}_{4}\right)$, filtration and evaporation of the solvent, the crude reaction mixture of $4 \mathbf{c}-\mathbf{e}$ or 13 was obtained. All crude reaction mixtures were purified by flash chromatography on silica gel.

\section{1-Allyl-4-benzyloxy-3-chloro-2,2-dimethylpyrrolidine 4a}

Colourless oil, $46 \%$ yield, TLC Rf $0.15\left(\mathrm{CH}_{2} \mathrm{Cl}_{2}\right) .{ }^{1} \mathbf{H}$ NMR $\left(300 \mathrm{MHz}, \mathrm{CDCl}_{3}\right): \delta 1.07$ and $1.15(6 \mathrm{H}, 2 \times \mathrm{s}), 2.53(1 \mathrm{H}, \mathrm{d} \times \mathrm{d}, J=10.5 \mathrm{~Hz}, J=4.1 \mathrm{~Hz}), 2.84(1 \mathrm{H}, \mathrm{d} \times \mathrm{d}, J=13.2 \mathrm{~Hz}$, $J=7.7 \mathrm{~Hz}), 3.35(1 \mathrm{H}, \mathrm{d} \times \mathrm{d}, J=13.2 \mathrm{~Hz}, J=5.2 \mathrm{~Hz}), 3.39(1 \mathrm{H}, \mathrm{d} \times \mathrm{d}, J=10.5 \mathrm{~Hz}, J=7.4$ $\mathrm{Hz}), 3.87(1 \mathrm{H}, \mathrm{d}, J=6.9 \mathrm{~Hz}), 4.02(1 \mathrm{H}, \mathrm{d} \times \mathrm{t}, J=6.9 \mathrm{~Hz}, J=3.9 \mathrm{~Hz}), 4.58(1 \mathrm{H}, \mathrm{d}, J=12.1$ 
$\mathrm{Hz}), 4.74(1 \mathrm{H}, \mathrm{d}, J=12.1 \mathrm{~Hz}), 5.05-5.22(2 \mathrm{H}, \mathrm{m}), 5.72-5.86(1 \mathrm{H}, \mathrm{m}), 7.26-7.40(5 \mathrm{H}, \mathrm{m})$.

${ }^{13}$ C NMR (75 MHz, $\left.\mathrm{CDCl}_{3}\right): \delta 15.0,25.0,52.1,57.1,63.0,68.4,72.6,75.0,116.7,127.5$, 127.7, 128.3, 136.4, 138.3. IR $\left(\mathrm{NaCl}, \mathrm{cm}^{-1}\right): v_{\max }=3066,3030,2971,2928,2869,2814$, 1721, 1643, 1497, 1454, 1383, 1365. MS (70 eV) m/z (\%): 380/2 $\left(\mathrm{M}^{+}+1,100\right)$. Anal. Calcd for $\mathrm{C}_{16} \mathrm{H}_{22} \mathrm{ClNO}$ : C 68.68; H 7.93; N 5.01. Found: C 68.82; H 7.82; N 5.16.

\section{1-Allyl-3-chloro-4-methoxy-2,2-dimethylpyrrolidine 4b}

Colourless oil, 44\% yield, TLC Rf 0.22 (petrol ether/ethyl acetate 10/1). ${ }^{1} \mathbf{H}$ NMR (300 $\left.\mathrm{MHz}, \mathrm{CDCl}_{3}\right): \delta 1.02$ and $1.14(6 \mathrm{H}, 2 \times \mathrm{s}), 2.46(1 \mathrm{H}, \mathrm{d} \times \mathrm{d}, J=10.5 \mathrm{~Hz}, J=3.6 \mathrm{~Hz}), 2.84$ $(1 \mathrm{H}, \mathrm{d} \times \mathrm{d}, J=13.2 \mathrm{~Hz}, J=7.4 \mathrm{~Hz}), 3.33(1 \mathrm{H}, \mathrm{d} \times \mathrm{d} \times \mathrm{t}, J=13.2 \mathrm{~Hz}, J=5.2 \mathrm{~Hz}, J=1.7 \mathrm{~Hz})$, $3.38(1 \mathrm{H}, \mathrm{d} \times \mathrm{d}, J=10.5 \mathrm{~Hz}, J=6.6 \mathrm{~Hz}), 3.40(3 \mathrm{H}, \mathrm{s}), 3.81(1 \mathrm{H}, \mathrm{d} \times \mathrm{t}, J=6.7 \mathrm{~Hz}, J=3.9$ $\mathrm{Hz}), 3.86(1 \mathrm{H}, \mathrm{d}, J=6.7 \mathrm{~Hz}), 5.06-5.23(2 \mathrm{H}, \mathrm{m}), 5.73-5.87(1 \mathrm{H}, \mathrm{m}) .{ }^{13} \mathbf{C}$ NMR $(75 \mathrm{MHz}$, $\left.\mathrm{CDCl}_{3}\right): \delta 15.1,24.9,52.1,56.7,58.6,62.9,68.1,77.6,116.7,136.4$. IR $\left(\mathrm{NaCl}, \mathrm{cm}^{-1}\right)$ : $v_{\max }=2973,2929,1643,1462,1383,1365$. MS $(70 \mathrm{eV}) \mathrm{m} / \mathrm{z}(\%): 204 / 6\left(\mathrm{M}^{+}+1,100\right)$. Anal. Calcd for $\mathrm{C}_{10} \mathrm{H}_{18} \mathrm{ClNO}$ : C 58.96; H 8.91; N 6.88. Found: C 59.14; H 8.78; N 6.98.

\section{4-Benzyloxy1-tert-butyl-2,2-dimethylpyrrolidin-3-ol 4c}

Colourless oil, 46\% yield, TLC Rf $0.1\left(\mathrm{CH}_{2} \mathrm{Cl}_{2} / \mathrm{MeOH} 20 / 1\right) .{ }^{1} \mathbf{H}$ NMR $(300 \mathrm{MHz}$, $\left.\mathrm{CDCl}_{3}\right): \delta 0.94$ and $1.08(6 \mathrm{H}, 2 \times \mathrm{s}), 2.53(1 \mathrm{H}, \mathrm{d} \times \mathrm{d}, J=10.7 \mathrm{~Hz}, J=3.9 \mathrm{~Hz}), 2.88(1 \mathrm{H}$, $\mathrm{d} \times \mathrm{d}, J=13.2 \mathrm{~Hz}, J=7.2 \mathrm{~Hz}), 3.23(1 \mathrm{H}, \mathrm{d} \times \mathrm{d}, J=10.7 \mathrm{~Hz}, J=7.7 \mathrm{~Hz}), 3.24(1 \mathrm{H}, \mathrm{d} \times \mathrm{d}, J=$ $13.2 \mathrm{~Hz}, J=5.8 \mathrm{~Hz}), 3.68(1 \mathrm{H}, \mathrm{d}, J=5.8 \mathrm{~Hz}), 4.03(1 \mathrm{H}, \mathrm{d} \times \mathrm{d} \times \mathrm{d}, J=7.2, J=7.2 \mathrm{~Hz}, J=$ $3.9 \mathrm{~Hz}), 4.48(1 \mathrm{H}, \mathrm{d}, J=11.6 \mathrm{~Hz}), 4.58(1 \mathrm{H}, \mathrm{d}, J=11.6 \mathrm{~Hz}), 5.05-5.22(2 \mathrm{H}, \mathrm{m}), 5.75-$ $5.89(1 \mathrm{H}, \mathrm{m}), 7.26-7.39(5 \mathrm{H}, \mathrm{m}) .{ }^{13} \mathrm{C}$ NMR $\left(75 \mathrm{MHz}, \mathrm{CDCl}_{3}\right): \delta 14.5,23.9,51.6,56.1$, 
$62.2,72.3,75.0,78.3,116.5,127.7,127.9,128.5,136.7,137.7$. IR $\left(\mathrm{NaCl}, \mathrm{cm}^{-1}\right): v_{\mathrm{OH}}=$ 3532; $v_{\max }=2965,2926,2870,2807,1642,1455$. MS $(70 \mathrm{eV}) \mathrm{m} / \mathrm{z}(\%): 262\left(\mathrm{M}^{+}+1,100\right)$. Anal. Calcd for $\mathrm{C}_{17} \mathrm{H}_{27} \mathrm{NO}_{2}$ : C 73.61; H 9.81; N 5.05. Found: C 73.75; H 9.93; N 4.89.

\section{1-Allyl-4-benzyloxy-2,2-dimethyl-3-pyrrolidinecarbonitrile 4d}

Colourless oil, 44\% yield, TLC Rf $0.15\left(\mathrm{CH}_{2} \mathrm{Cl}_{2} / \mathrm{MeOH}\right.$ 99/1). ${ }^{1} \mathbf{H}$ NMR (300 MHz, $\left.\mathrm{CDCl}_{3}\right): \delta 1.12$ and $1.27(6 \mathrm{H}, 2 \times \mathrm{s}), 2.87(1 \mathrm{H}, \mathrm{d} \times \mathrm{d}, J=13.4 \mathrm{~Hz}, J=7.0 \mathrm{~Hz}), 2.94-3.05$ $(2 \mathrm{H}, \mathrm{m}), 3.15-3.24(3 \mathrm{H}, \mathrm{m}), 4.69(2 \mathrm{H}, \mathrm{s}), 5.11-5.25(2 \mathrm{H}, \mathrm{m}), 5.70-5.83(1 \mathrm{H}, \mathrm{m}), 7.27-$ $7.40(5 \mathrm{H}, \mathrm{m}) .{ }^{13} \mathrm{C}$ NMR $\left(75 \mathrm{MHz}, \mathrm{CDCl}_{3}\right): \delta 17.3,23.9,29.1,45.3,50.5,52.9,63.3$, $65.4,117.5,119.4,127.0,127.7,128.6,135.1,140.9 . \mathbf{I R}\left(\mathrm{NaCl}, \mathrm{cm}^{-1}\right): v_{\mathrm{C} \equiv \mathrm{N}}=2246 ; v_{\max }=$ 2972, 2927, 2851, 1720, 1624, 1454. MS (70 eV) m/z (\%): $271\left(\mathrm{M}^{+}+1,100\right)$. Anal. Calcd for $\mathrm{C}_{17} \mathrm{H}_{22} \mathrm{~N}_{2} \mathrm{O}$ : C 75.52; $\mathrm{H} 8.20 ; \mathrm{N} 10.36$. Found: C 75.62; H 8.10; N 10.14 .

\section{1-Allyl-3-azido-4-benzyloxy-2,2-dimethylpyrrolidine 4e}

Colourless oil, 52\% yield, TLC Rf 0.25 (petroleum ether/ethyl acetate 4/1). ${ }^{1} \mathbf{H}$ NMR (300 MHz, $\left.\mathrm{CDCl}_{3}\right): \delta 1.05$ and $1.12(6 \mathrm{H}, 2 \times \mathrm{s}), 2.73(1 \mathrm{H}, \mathrm{d} \times \mathrm{d}, J=10.7 \mathrm{~Hz}, J=4.4 \mathrm{~Hz})$, $2.99(1 \mathrm{H}, \mathrm{d} \times \mathrm{d}, J=13.4 \mathrm{~Hz}, J=6.5 \mathrm{~Hz}), 3.10(1 \mathrm{H}, \mathrm{d} \times \mathrm{d} \times \mathrm{t}, J=13.4 \mathrm{~Hz}, J=6.1 \mathrm{~Hz}, J=1.4$ $\mathrm{Hz}), 3.14(1 \mathrm{H}, \mathrm{d} \times \mathrm{d}, J=10.7 \mathrm{~Hz}, J=7.7 \mathrm{~Hz}), 3.32(1 \mathrm{H}, \mathrm{d}, J=6.6 \mathrm{~Hz}), 4.21(1 \mathrm{H}, \mathrm{d} \times \mathrm{d} \times \mathrm{d}, J$ $=7.7, J=6.6 \mathrm{~Hz}, J=4.4 \mathrm{~Hz}), 4.54(1 \mathrm{H}, \mathrm{d}, J=12.1 \mathrm{~Hz}), 4.63(1 \mathrm{H}, \mathrm{d}, J=12.1 \mathrm{~Hz}), 5.04-$ $5.22(2 \mathrm{H}, \mathrm{m}), 5.73-5.86(1 \mathrm{H}, \mathrm{m}), 7.25-7.38(5 \mathrm{H}, \mathrm{m}) .{ }^{13} \mathbf{C} \mathbf{N M R}\left(75 \mathrm{MHz}, \mathrm{CDCl}_{3}\right): \delta 17.7$, $23.1,51.2,56.5,63.5,70.1,72.2,75.9,116.4,127.6,127.7,128.4,136.5,137.9$. IR $\left(\mathrm{NaCl}, \mathrm{cm}^{-1}\right): v_{\mathrm{N} 3}=2105 ; v_{\max }=2969,2926,2871,2811,1643,1455 . \mathbf{M S}(70 \mathrm{eV}) \mathrm{m} / \mathrm{z}$ 
(\%): $287\left(\mathrm{M}^{+}+1,100\right)$. Anal. Calcd for $\mathrm{C}_{16} \mathrm{H}_{22} \mathrm{~N}_{4} \mathrm{O}$ : C 67.11; H 7.74; N 19.56. Found: $\mathrm{C}$ 67.22; H 7.85; N 19.36 .

Cis-5-benzyloxy-4-bromo-1-t-butyl-3,3-dimethylpiperidine 12a

Colourless oil, $98 \%$ yield. ${ }^{1} \mathbf{H}$ NMR $\left(300 \mathrm{MHz}, \mathrm{CDCl}_{3}\right): \delta 1.03$ and $1.13(6 \mathrm{H}, 2 \times \mathrm{s}), 2.18$ $2.25(1 \mathrm{H}, \mathrm{m}), 2.40-2.50(1 \mathrm{H}, \mathrm{m}), 2.45-2.80(2 \mathrm{H}, \mathrm{m}), 3.65-3.72(1 \mathrm{H}, \mathrm{m}), 4.27-4.32(1 \mathrm{H}$, m), $4.47(1 \mathrm{H}, \mathrm{d}, J=11.4 \mathrm{~Hz}), 4.65(1 \mathrm{H}, \mathrm{d}, J=11.4 \mathrm{~Hz}), 7.24-7.41(5 \mathrm{H}, \mathrm{m}) .{ }^{13} \mathbf{C}$ NMR $\left(75 \mathrm{MHz}, \mathrm{ref}=\mathrm{CDCl}_{3}\right): \delta 26.4,27.1,36.3,46.5,46.5,53.4,67.8,70.5,74.8,127.7$, 127.9, 128.4, 128.6, 138.3. IR $\left(\mathrm{NaCl}, \mathrm{cm}^{-1}\right): v_{\max }=2974,2892,2812,1477,1454,1390$, 1370, 1359, 1339, 1256, 1226, 1200, 1138, 1104, 1025, 1011. MS (70 eV): m/z (\%) 354/6 (M+1, 100); 343 (12); 294 (9); 293 (50); 264 (18); 263 (87). Anal. Calcd for $\mathrm{C}_{18} \mathrm{H}_{28} \mathrm{BrNO}$ : C 61.02; H 7.97; N 3.95. Found: C 60.90; H 8.12; N 4.09.

\section{Cis-1-benzyl-5-benzyloxy-4-bromo-3,3-dimethylpiperidine 12b}

Colourless oil, $97 \%$ yield. ${ }^{1}$ H NMR (300 MHz, $\left.\mathrm{CDCl}_{3}\right): \delta 1.12$ and $1.16(6 \mathrm{H}, 2 \times \mathrm{s}), 2.05-$ $2.45(2 \mathrm{H}, \mathrm{m}), 2.45-2.75(2 \mathrm{H}, \mathrm{m}), 3.44(1 \mathrm{H}, \mathrm{d}, J=13.5 \mathrm{~Hz}), 3.54(1 \mathrm{H}, \mathrm{d}, J=13.5 \mathrm{~Hz})$ 3.71-3.81 (1H, m), 4.19-4.30 (1H, m), $4.43(1 \mathrm{H}, \mathrm{d}, J=11.8 \mathrm{~Hz}), 4.60(1 \mathrm{H}, \mathrm{d}, J=11.8$ $\mathrm{Hz})$, 7.19-7.37 $(10 \mathrm{H}, \mathrm{m}) .{ }^{13} \mathbf{C}$ NMR $\left(75 \mathrm{MHz}, \mathrm{ref}=\mathrm{CDCl}_{3}\right): \delta 27.8,29.2,36.7,53.7$, $60.5,62.3,67.1,70.5,74.8,127.1,127.8,127.9,128.4,128.5,128.8,138.3,138.9$. IR $\left(\mathrm{NaCl}, \mathrm{cm}^{-1}\right): v_{\max }=2956,2926,2869,2817,1495,1466,1454,1366,1109,1027$. MS (70 eV): m/z (\%) 388/90 (M+1, 100); 387 (39); 312 (17); 311 (56). Anal. Calcd for $\mathrm{C}_{21} \mathrm{H}_{26} \mathrm{BrNO}$ : C 64.95; H 6.75; N 3.61. Found: C 65.12; H 6.63; N 3.53. 


\section{Cis-1-benzyl-4-bromo-5-methoxy-3,3-dimethylpiperidine 12c}

Colourless oil, 92\% yield. ${ }^{1} \mathbf{H}$ NMR (300 MHz, $\left.\mathrm{CDCl}_{3}\right): \delta 1.10$ and $1.25(6 \mathrm{H}, 2 \times \mathrm{s}), 2.09$ $2.18(1 \mathrm{H}, \mathrm{m}), 2.30-2.40(1 \mathrm{H}, \mathrm{m}), 2.50-2.82(2 \mathrm{H}, \mathrm{m}), 3.35(3 \mathrm{H}, \mathrm{s}), 3.50(1 \mathrm{H}, \mathrm{d}, J=13.4$ $\mathrm{Hz}), 3.53-3.59(1 \mathrm{H}, \mathrm{m}) ; 3.59(1 \mathrm{H}, \mathrm{d}, J=13.4 \mathrm{~Hz}), 4.21-4.32(1 \mathrm{H}, \mathrm{m}), 7.23-7.32(5 \mathrm{H}, \mathrm{m})$.

${ }^{13}$ C NMR $\left(75 \mathrm{MHz}\right.$, ref $\left.=\mathrm{CDCl}_{3}\right): \delta 26.9,29.0,36.5,52.8,56.3,58.8,62.3,66.7,75.4$, 127.2, 128.4, 128.8, 138.6. IR $\left(\mathrm{NaCl}, \mathrm{cm}^{-1}\right): v_{\max }=2955,2927,2872,2818,2787,1466$, 1454, 1365, 1341, 1221, 1201, 1115, 1026. MS (70 eV): m/z (\%) 312/4 (M+1, 100); 296 (7); 232/234 (13). Anal. Calcd for $\mathrm{C}_{15} \mathrm{H}_{22} \mathrm{BrNO}$ : C 57.70; $\mathrm{H} 7.10 ; \mathrm{N} 4.49$. Found: $\mathrm{C}$ $57.52 ; \mathrm{H} 7.21 ; \mathrm{N} 4.63$.

\section{Cis-1-allyl-5-benzyloxy-4-bromo-3,3-dimethylpiperidine 12d}

Colourless oil, $95 \%$ yield. ${ }^{1} \mathbf{H}$ NMR $\left(300 \mathrm{MHz}, \mathrm{CDCl}_{3}\right): \delta 1.13$ and $1.16(6 \mathrm{H}, 2 \times \mathrm{s}), 2.05-$ $2.40(2 \mathrm{H}, \mathrm{m}), 2.45-2.75(2 \mathrm{H}, \mathrm{m}), 2.94(1 \mathrm{H}, \mathrm{d} \times \mathrm{d}, J=13.6 \mathrm{~Hz}, J=6.5 \mathrm{~Hz}), 3.01(1 \mathrm{H}, \mathrm{d} \times \mathrm{d}$, $J=13.6 \mathrm{~Hz}, J=6.3 \mathrm{~Hz}), 3.70-3.74(1 \mathrm{H}, \mathrm{m}), 4.20-4.31(1 \mathrm{H}, \mathrm{m}), 4.48(1 \mathrm{H}, \mathrm{d}, J=11.8 \mathrm{~Hz})$, $4.64(1 \mathrm{H}, \mathrm{d}, J=11.8 \mathrm{~Hz}), 5.09-5.20(2 \mathrm{H}, \mathrm{m}), 5.72-5.86(1 \mathrm{H}, \mathrm{m}), 7.23-7.39(5 \mathrm{H}, \mathrm{m}) .{ }^{13} \mathrm{C}$ NMR $\left(75 \mathrm{MHz}, \mathrm{ref}=\mathrm{CDCl}_{3}\right): \delta 27.1,28.8,36.5,53.7,59.8,61.2,67.0,70.6,73.4,117.7$, 127.4, 127.8, 127.9, 128.5, 135.4, 138.2. IR $\left(\mathrm{NaCl}, \mathrm{cm}^{-1}\right): v_{\max }=2958,2928,2906,2870$, 2806, 1455, 1366, 1109, 1028. MS (70 eV): m/z (\%) 338/40 (M+1, 67); 263 (10); 262 (23); 261 (100). Anal. Calcd for $\mathrm{C}_{17} \mathrm{H}_{24} \mathrm{BrNO}$ : C 60.36; H 7.15; N 4.14. Found: C 60.50; H 7.04; N 4.01 . 


\section{Cis-5-benzyloxy-4-bromo-1-cyclohexyl-3,3-dimethylpiperidine 12e}

Colourless oil, $95 \%$ yield. ${ }^{1}$ H NMR (300 MHz, $\left.\mathrm{CDCl}_{3}\right): \delta 1.12$ and $1.14(6 \mathrm{H}, 2 \times \mathrm{s}), 1.57-$ $1.76(10 \mathrm{H}, \mathrm{m}), 2.10(1 \mathrm{H}, \mathrm{d}, J=11.3 \mathrm{~Hz}), 2.25-2.29(1 \mathrm{H}, \mathrm{m}), 2.55(1 \mathrm{H}, \mathrm{d}, J=11.3 \mathrm{~Hz})$, 2.55-2.80 (2H, m), 3.61-3.70 (1H, m), 4.19-4.28 (1H, m), $4.45(1 \mathrm{H}, \mathrm{d}, J=11.8 \mathrm{~Hz}), 4.61$ $(1 \mathrm{H}, \mathrm{d}, J=11.8 \mathrm{~Hz}), 7.19-7.38(5 \mathrm{H}, \mathrm{m}) .{ }^{13} \mathbf{C}$ NMR $\left(75 \mathrm{MHz}, \mathrm{ref}=\mathrm{CDCl}_{3}\right): \delta 26.0,26.1$, $26.6,27.1,28.1,28.7,29.2,36.4,50.1,55.6,63.4,67.9,70.4,74.3,127.6,127.7,128.4$, 138.4. IR $\left(\mathrm{NaCl}, \mathrm{cm}^{-1}\right): v_{\max }=2928,2854,1453,1382,1364,1343,1210,1109,1028$. MS (70 eV): m/z (\%) 380/2 ( $\left.\mathrm{M}^{+}+1,100\right)$. Anal. Calcd for $\mathrm{C}_{20} \mathrm{H}_{30} \mathrm{BrNO}$ : C 63.15; H 7.95; N 3.68. Found: C 63.28; H 7.82; N 3.74.

\section{Cis-1-allyl-5-benzyloxy-3,3-dimethylpiperidin-4-ol 14a}

Colourless oil, $86 \%$ yield. ${ }^{1} \mathbf{H}$ NMR $\left(300 \mathrm{MHz}, \mathrm{CDCl}_{3}\right): \delta 0.99(6 \mathrm{H}, \mathrm{s}), 2.13(2 \mathrm{H}, \mathrm{s})$, 2.17-2.37 (1H, m), 2.67-2.76 (1H, m), $2.94(1 \mathrm{H}, \mathrm{d} \times \mathrm{d}, J=13.6 \mathrm{~Hz}, J=6.8 \mathrm{~Hz}), 3.01(1 \mathrm{H}$, $\mathrm{d} \times \mathrm{d}, J=13.6 \mathrm{~Hz}, J=6.1 \mathrm{~Hz}), 3.52(1 \mathrm{H}, \mathrm{d}, J=2.8 \mathrm{~Hz}), 3.76-3.82(1 \mathrm{H}, \mathrm{m}), 4.55(1 \mathrm{H}, \mathrm{d}, J$ $=12.1 \mathrm{~Hz}), 4.59(1 \mathrm{H}, \mathrm{d}, J=12.1 \mathrm{~Hz}), 5.09-5.19(2 \mathrm{H}, \mathrm{m}), 5.74-5.88(1 \mathrm{H}, \mathrm{m}), 7.24-7.37$ $(5 \mathrm{H}, \mathrm{m}) .{ }^{13} \mathrm{C}$ NMR $\left(75 \mathrm{MHz}, \mathrm{CDCl}_{3}\right): \delta 24.1,24.8,35.3,51.9,59.2,61.3,70.7,72.9$, $74.8,117.3,127.6,127.8,128.4,135.5,138.1 . \mathbf{I R}\left(\mathrm{NaCl}, \mathrm{cm}^{-1}\right): v_{\mathrm{OH}}=3561 . \mathbf{M S}(70 \mathrm{eV})$ : m/z (\%) $276\left(100, \mathrm{M}^{+}+1\right)$. Anal. Calcd for $\mathrm{C}_{17} \mathrm{H}_{25} \mathrm{NO}_{2}$ : C 74.14; H 9.15; N 5.09. Found: C 74.01; H 9.29; N 4.94.

\section{Cis-1-allyl-5-benzyloxy-3,3-dimethyl-4-piperidinecarbonitrile 14b}

Colourless oil, $92 \%$ yield. ${ }^{1} \mathbf{H}$ NMR $\left(300 \mathrm{MHz}, \mathrm{CDCl}_{3}\right): \delta 1.07$ and $1.16(6 \mathrm{H}, 2 \times \mathrm{s}), 2.10-$ $2.15(1 \mathrm{H}, \mathrm{m}), 2.20-2.38(2 \mathrm{H}, \mathrm{m}), 2.76(1 \mathrm{H}, \mathrm{d}, J=3.3 \mathrm{~Hz}), 2.85-3.06(3 \mathrm{H}, \mathrm{m}), 3.79-3.83$ 
$(1 \mathrm{H}, \mathrm{m}), 4.56-4.64(2 \mathrm{H}, \mathrm{m}), 5.12-5.20(2 \mathrm{H}, \mathrm{m}), 5.71-5.84(1 \mathrm{H}, \mathrm{m}), 7.26-7.36(5 \mathrm{H}, \mathrm{m})$.

${ }^{13} \mathrm{C}$ NMR (75 MHz, $\left.\mathrm{CDCl}_{3}\right): \delta 26.1,26.8,34.0,43.9,54.5,61.0,71.0,71.3,117.9,118.2$, 127.6, 127.8, 128.5, 134.9, 137.7. IR $\left(\mathrm{NaCl}, \mathrm{cm}^{-1}\right): v_{\mathrm{CN}}=2239 . \mathbf{M S}(70 \mathrm{eV}): \mathrm{m} / \mathrm{z}(\%) 285$ $\left(\mathrm{M}^{+}+1,100\right)$. Anal. Calcd for $\mathrm{C}_{18} \mathrm{H}_{24} \mathrm{~N}_{2} \mathrm{O}$ : C 76.02; $\mathrm{H} 8.51 ; \mathrm{N}$ 9.85. Found: $\mathrm{C} 75.88 ; \mathrm{H}$ $8.73 ; \mathrm{N} 9.80$.

\section{Cis-1-allyl-4-azido-5-benzyloxy-3,3-dimethylpiperidine 14c}

Colourless oil, $72 \%$ yield. ${ }^{1} \mathbf{H}$ NMR (300 MHz, $\left.\mathrm{CDCl}_{3}\right): \delta 0.97$ and $1.03(6 \mathrm{H}, 2 \times \mathrm{s}), 1.94$ $(1 \mathrm{H}, \mathrm{d}, J=11.4 \mathrm{~Hz}), 2.12(1 \mathrm{H}, \mathrm{d}, J=11.4 \mathrm{~Hz}), 2.21-2.30(1 \mathrm{H}, \mathrm{m}), 2.72-2.87(1 \mathrm{H}, \mathrm{m})$, $2.91(1 \mathrm{H}, \mathrm{d} \times \mathrm{d}, J=13.5 \mathrm{~Hz}, J=6.8 \mathrm{~Hz}), 3.00(1 \mathrm{H}, \mathrm{d} \times \mathrm{d}, J=13.5 \mathrm{~Hz}, J=6.1 \mathrm{~Hz}), 3.42-$ $3.45(1 \mathrm{H}, \mathrm{m}), 3.90-3.96(1 \mathrm{H}, \mathrm{m}), 4.60(2 \mathrm{H}, \mathrm{s}), 5.08-5.18(2 \mathrm{H}, \mathrm{m}), 5.71-5.84(1 \mathrm{H}, \mathrm{m})$, 7.22-7.37 (5H, m). ${ }^{13} \mathrm{C}$ NMR (75 MHz, $\left.\mathrm{CDCl}_{3}\right): \delta 25.4,25.7,35.5,52.5,59.0,61.1,68.7$, $71.0,74.9,117.4,127.4,127.6,128.4,135.3,138.2 . \mathbf{I R}\left(\mathrm{NaCl}, \mathrm{cm}^{-1}\right): v_{\mathrm{N} 3}=2102 . \mathbf{M S}(70$ eV): m/z (\%) $301\left(\mathrm{M}^{+}+1,100\right) ; 246$ (12). Anal. Calcd for $\mathrm{C}_{17} \mathrm{H}_{24} \mathrm{~N}_{4} \mathrm{O}: \mathrm{C}$ 67.97; H 8.05; N 18.65. Found: C 67.90; H 8.19; N 18.49.

\section{Synthesis of 3-bromopyrrolidines 7 from 2-(hydroxyethyl)aziridines 6}

As a representative example, the synthesis of 1-allyl-4-benzyloxy-3-bromo-2,2dimethylpyrrolidine 7a is described. To a solution of 2-(1-allyl-3,3-dimethylaziridin-2yl)-2-benzyloxyethanol 6a $(0.5 \mathrm{~g}, 1.92 \mathrm{mmol})$ in $30 \mathrm{ml}$ of THF was added $\mathrm{N}$ bromosuccinimide $(0.41 \mathrm{~g}, 2.23 \mathrm{mmol})$ and triphenylphosphine $(0.60 \mathrm{~g}, 2.23 \mathrm{mmol})$. After stirring at room temperature for 18 hours, $30 \mathrm{ml}$ water was added, and the mixture was extracted three times with $30 \mathrm{ml}$ of diethyl ether. The combined organic fractions were 
dried $\left(\mathrm{MgSO}_{4}\right)$ and, after filtration and evaporation of the solvent, the crude reaction mixture was obtained. Purification was performed by flash chromatography on silica gel.

\section{1-Allyl-4-benzyloxy-3-bromo-2,2-dimethylpyrrolidine 7a}

Colourless oil, $68 \%$ yield, TLC Rf $0.15\left(\mathrm{CH}_{2} \mathrm{Cl}_{2} / \mathrm{MeOH}\right.$ 99/1). ${ }^{1} \mathrm{H}$ NMR $(300 \mathrm{MHz}$, $\left.\mathrm{CDCl}_{3}\right): \delta 1.11$ and $1.15(6 \mathrm{H}, 2 \times \mathrm{s}), 2.54(1 \mathrm{H}, \mathrm{d} \times \mathrm{d}, J=10.5 \mathrm{~Hz}, J=3.0 \mathrm{~Hz}), 2.85(1 \mathrm{H}$, $\mathrm{d} \times \mathrm{d}, J=13.2 \mathrm{~Hz}, J=7.7 \mathrm{~Hz}), 3.34-3.44(2 \mathrm{H}, \mathrm{m}), 3.96(2 \mathrm{H}, \mathrm{m}), 4.56(1 \mathrm{H}, \mathrm{d}, J=12.1)$, $4.71(1 \mathrm{H}, \mathrm{d}, J=12.1 \mathrm{~Hz}), 5.06-5.22(2 \mathrm{H}, \mathrm{m}), 5.72-5.86(1 \mathrm{H}, \mathrm{m}), 7.24-7.38(5 \mathrm{H}, \mathrm{m}) .{ }^{13} \mathrm{C}$ NMR $\left(75 \mathrm{MHz}\right.$, ref $\left.=\mathrm{CDCl}_{3}\right): \delta 16.4,25.2,52.6,57.4,62.1,62.7,72.6,75.1,116.8$, 127.5, 127.6, 128.5, 136.6, 138.4. IR $\left(\mathrm{NaCl}, \mathrm{cm}^{-1}\right): v_{\max }=3066,3030,2971,2927,2869$, 2814, 1721, 1643, 1497, 1455, 1383, 1365. MS (70 eV) m/z (\%): 324/6 $\left(\mathrm{M}^{+}+1,100\right)$. Anal. Calcd for $\mathrm{C}_{16} \mathrm{H}_{22} \mathrm{BrNO}$ : C 59.27; H 6.84; N 4.32. Found: C 59.39; H 6.96; N 4.20.

\section{1-Allyl-3-bromo-4-methoxy-2,2-dimethylpyrrolidine 7b}

Colourless oil, 63\% yield, TLC Rf $0.45\left(\mathrm{CH}_{2} \mathrm{Cl}_{2} / \mathrm{MeOH} 33 / 1\right) .{ }^{1} \mathrm{H}$ NMR $(270 \mathrm{MHz}$, $\left.\mathrm{CDCl}_{3}\right): \delta 1.06$ and $1.14(6 \mathrm{H}, 2 \times \mathrm{s}), 2.48(1 \mathrm{H}, \mathrm{d} \times \mathrm{d}, J=10.4 \mathrm{~Hz}, J=3.8 \mathrm{~Hz}), 2.85(1 \mathrm{H}$, $\mathrm{d} \times \mathrm{d}, J=13.2 \mathrm{~Hz}, J=7.6 \mathrm{~Hz}), 3.33-3.43(2 \mathrm{H}, \mathrm{m}), 3.39(3 \mathrm{H}, \mathrm{s}), 3.76(1 \mathrm{H}, \mathrm{d} \times \mathrm{t}, J=6.8 \mathrm{~Hz}$, $J=4.0 \mathrm{~Hz}), 3.95(1 \mathrm{H}, \mathrm{d}, J=6.8 \mathrm{~Hz}), 5.07-5.23(2 \mathrm{H}, \mathrm{m}), 5.73-5.88(1 \mathrm{H}, \mathrm{m}) .{ }^{13} \mathbf{C} \mathbf{N M R}$ $\left(75 \mathrm{MHz}\right.$, ref $\left.=\mathrm{CDCl}_{3}\right): \delta 16.3,24.9,52.3,56.7,58.3,61.6,62.3,77.3,116.4,136.4 . \mathbf{I R}$ $\left(\mathrm{NaCl}, \mathrm{cm}^{-1}\right): v_{\max }=3078,2972,2928,2826,1722,1643,1462,1383,1366$. MS $(70 \mathrm{eV})$ m/z (\%): 248/50 $\left(\mathrm{M}^{+}+1,100\right)$. Anal. Calcd for $\mathrm{C}_{10} \mathrm{H}_{18} \mathrm{BrNO}$ : C 48.40; $\mathrm{H}$ 7.31; $\mathrm{N}$ 5.64. Found: C 48.65; H 7.42; N 5.47. 


\section{Synthesis of 2,5-dihydro-1H-pyrroles 8}

As a representative example, the synthesis of 1-allyl-4-benzyloxy-2,2-dimethyl-2,5dihydro- $1 H$-pyrrole 8a is described. Sodium hydride $(0.074 \mathrm{~g}, 3.0 \mathrm{mmol})$ was added to 30 $\mathrm{ml}$ of DMSO. After all of the sodium hydride was reacted, 1-allyl-4-benzyloxy-3-bromo2,2-dimethylpyrrolidine $7 \mathbf{a}(0.5 \mathrm{~g}, 1.5 \mathrm{mmol})$ was added and the reaction mixture was stirred for 2 hours at $130^{\circ} \mathrm{C}$. After two hours, the reaction mixture was cooled to room temperature and $30 \mathrm{ml}$ of water was added. The resulting suspension was extracted three times with $30 \mathrm{ml}$ of diethyl ether, and the combined organic fractions were dried $\left(\mathrm{MgSO}_{4}\right)$. After filtration and evaporation of the solvent 1-allyl-4-benzyloxy-2,2dimethyl-2,5-dihydro-1 $1 H$-pyrrole 8a was obtained.

\section{1-Allyl-4-benzyloxy-2,2-dimethyl-2,5-dihydro-1H-pyrrole 8a}

Colourless oil, 88\% yield. ${ }^{1} \mathbf{H}$ NMR (300 MHz, $\left.\mathrm{CDCl}_{3}\right): \delta 1.14(6 \mathrm{H}, \mathrm{s}), 3.15(2 \mathrm{H}, \mathrm{d} \times \mathrm{t}, J=$ $6.3 \mathrm{~Hz}, J=1.2 \mathrm{~Hz}), 3.45(2 \mathrm{H}, \mathrm{d}, J=1.4 \mathrm{~Hz}), 4.58(1 \mathrm{H}, \mathrm{t}, J=1.2 \mathrm{~Hz}), 4.76(2 \mathrm{H}, \mathrm{s}), 5.04-$ $5.24(1 \mathrm{H}, \mathrm{m}), 5.82-5.99(1 \mathrm{H}, \mathrm{m}), 7.27-7.46(5 \mathrm{H}, \mathrm{m}) .{ }^{13} \mathbf{C}$ NMR $\left(75 \mathrm{MHz}, \mathrm{CDCl}_{3}\right): \delta 24.8$, $51.5,56.5,64.8,71.2,104.3,116.3,127.7,128.0,128.5,136.7,137.3,154.9$. IR $(\mathrm{NaCl}$, $\left.\mathrm{cm}^{-1}\right): v_{\max }=2958,2924,1656,1456,1360$. MS $(70 \mathrm{eV}) \mathrm{m} / \mathrm{z}(\%): 244\left(\mathrm{M}^{+}+1,100\right)$. Anal. Calcd for $\mathrm{C}_{16} \mathrm{H}_{21} \mathrm{NO}$ : C 78.97; H 8.70; N 5.76. Found: C 78.79; H 8.90; N 5.87.

\section{1-Allyl-4-methoxy-2,2-dimethyl-2,5-dihydro-1H-pyrrole 8b}

Colourless oil, $86 \%$ yield. ${ }^{1} \mathbf{H}$ NMR (300 MHz, $\left.\mathrm{CDCl}_{3}\right): \delta 1.12(6 \mathrm{H}, \mathrm{s}), 3.15(2 \mathrm{H}, \mathrm{d} \times \mathrm{t}, J=$ $6.5 \mathrm{~Hz}, J=1.4 \mathrm{~Hz}), 3.37(2 \mathrm{H}, \mathrm{d}, J=1.7 \mathrm{~Hz}), 3.58(3 \mathrm{H}, \mathrm{s}), 4.48(1 \mathrm{H}, \mathrm{t}, J=1.4 \mathrm{~Hz}), 5.05-$ $5.25(1 \mathrm{H}, \mathrm{m}), 5.82-5.95(1 \mathrm{H}, \mathrm{m}) .{ }^{13} \mathrm{C}$ NMR $\left(75 \mathrm{MHz}, \mathrm{CDCl}_{3}\right): \delta 24.9,51.5,56.3,56.4$, 
64.8, 103.2, 116.3, 137.4, 156.0. IR $\left(\mathrm{NaCl}, \mathrm{cm}^{-1}\right): v_{\max }=2924,1664,1459,1377 . \mathbf{M S}(70$ eV) m/z (\%): 168( $\left.\mathrm{M}^{+}+1,80\right) ; 136(100)$. Anal. Calcd for $\mathrm{C}_{10} \mathrm{H}_{17} \mathrm{NO}$ : C 71.81; H 10.25; N 8.37. Found: C 71.65; H 10.39; N 8.49.

\section{Synthesis of 1-allyl-5,5-dimethyl-3-pyrrolidinone 9}

1-Allyl-4-methoxy-2,2-dimethyl-2,5-dihydro- $1 H$-pyrrole $\mathbf{8 b}$ was dissolved in $30 \mathrm{ml}$ of $2 \mathrm{~N} \mathrm{HCl}$ and stirred at room temperature for 18 hours. After the reaction, $1 \mathrm{~N} \mathrm{NaOH}$ was added until the reaction mixture was basic. After extraction of this mixture with three times $30 \mathrm{ml}$ of dichloromethane, drying $\left(\mathrm{MgSO}_{4}\right)$ of the combined organic fractions, filtration and evaporation of the solvent, 1-allyl-5,5-dimethyl-3-pyrrolidinone 9 was obtained in $89 \%$ yield.

\section{1-Allyl-5,5-dimethyl-3-pyrrolidinone 9}

Colourless oil, 89\% yield. ${ }^{1} \mathbf{H}$ NMR $\left(300 \mathrm{MHz}, \mathrm{CDCl}_{3}\right): \delta 1.18(6 \mathrm{H}, \mathrm{s}), 2.31(2 \mathrm{H}, \mathrm{s}), 3.11$ $(2 \mathrm{H}, \mathrm{s}), 3.14(2 \mathrm{H}, \mathrm{d} \times \mathrm{d}, J=6.3 \mathrm{~Hz}, J=0.8 \mathrm{~Hz}), 5.09-5.25(2 \mathrm{H}, \mathrm{m}), 5.77-5.90(1 \mathrm{H}, \mathrm{m}) .{ }^{13} \mathrm{C}$ NMR (75 MHz, $\left.\mathrm{CDCl}_{3}\right): \delta 22.5,51.2,53.4,59.0,59.1,116.9,136.1,214.1$. IR $(\mathrm{NaCl}$, $\left.\mathrm{cm}^{-1}\right): v_{\max }=2969,2930,2872,2807,1758,1693,1367 . \mathrm{MS}(70 \mathrm{eV}) \mathrm{m} / \mathrm{z}(\%): 154\left(\mathrm{M}^{+}+1\right.$, 100). Anal. Calcd for $\mathrm{C}_{9} \mathrm{H}_{15} \mathrm{NO}$ : C 70.55; H 9.87; N 9.14. Found: C 70.69; H 9.97; N 9.02 . 